\title{
Gas-Sensing Properties of Noble Metal Decorated Carbon Nanotubes
}

\author{
$1,{ }^{*}$ Kleuton Antunes Lopes Lima (PG), ${ }^{2}$ Bernhard Georg Enders Neto (PQ) and ${ }^{3}$ Luiz \\ Antônio Ribeiro Júnior (PQ) \\ ${ }^{1}$ Institute of Physics, University of Brasília, Brasília, 70910-970, DF, Brazil, ${ }^{2}$ University of Brasília, PPG- \\ CIMA, Campus Planaltina, 73345-010, Brasília, DF, Brazil, ${ }^{3}$ International Center for Condensed Matter \\ Physics, University of Brasília, P.O. Box 04531, 70.919-970, Brasília, DF, Brazil \\ e-mail: kleuton.ufpi.2010-1@hotmail.com \\ Keywords: Carbon Nanotubes, Gas-sensing, Functionalization, Adsorption.
}

\section{Introduction}

Studies about carbon nanotubes have aroused great interest since their discovery in the beginning of 90's [1]. Due to their vast potential for several applications, nowadays researchers from different areas have employed a great effort to understand the fundamental properties governing their electronic structure and susceptibility towards chemical reaction [2]. Importantly, carbon nanotubes show high sensitivity towards changes in their local environment that stems from the susceptibility of their electronic structure to interacting molecules [2]. This chemical sensitivity has made them ideal candidates for incorporation into the design of chemical sensors. Despite the lengthy list of accomplishments, several key challenges must be addressed before carbon nanotubes are capable of competing with the current commercial technology for sensor materials. One of these challenges is, certainly, the obtation of processes that might enhance its sensing properties. Here, we theoretically study -- in the framework of Density Functional Theory (DFT) calculations -- how single-walled carbon nanotubes (SWCNTs) can have their sensing traits enhanced under functionalization with noble metals.

\section{Methodology}

The DFT calculations presented here were carried out using a commercial software package named Materials Studio, which is developed by Accelrys Inc. This software includes the module DMol3, which uses DFT with a numerical radial function basis set to calculate the electronic properties of molecules, clusters, surfaces and crystalline solid materials from first principles. Here we calculate the gas adsorption on noble metal decorated SWCNTs by using GGA/PBE method on DFT and the following gases: $\mathrm{CO} 2$ and NH3.

\section{Results}

Results of DFT calculations show that doped carbon nanotubes can respond to the presence of $\mathrm{CO} 2$ and NH3. Furthermore, the electrical characteristics of noble metal decorated SWCNTs show different degrees of changes after adsorption of the test gases. As $\mathrm{CO} 2 / \mathrm{NH} 3$ is adsorbed on doped SWCNTs, the CNTs lose electrons, the 
number of hole carriers is increased, and conductivity is enhanced. The figure bellow presents the electronic density distribution for the SWCNT@CO2 complex.

\section{Conclusions}

In this study, the adsorptions of the gases mentioned above on the surface of carbon nanotubes were studied. The gas-sensing properties of noble metal decorated carbon nanotubes were assessed according to the changes in geometric and electronic structures during adsorption. The main conclusion is that doped nanotubes effectively improves the adsorption sensitivity of intrinsic carbon nanotubes for all complexes studied here.

\section{Acknowledgements}

The authors gratefully acknowledge the financial support from the Brazilian Research Councils CAPES and FAPDF. L.A.R.J. gratefully acknowledges the financial support from the Brazilian Research Council FAPDF grant 0193.000942/2015 and 193.001.511/2017. L.A.R.J. also wishes to thank the Brazilian Ministry of Planning, Budget and Management (Grant DIPLA 005/2016).



Figure: Electronic density distribution.

\section{References}

[1] D. R. Kauffman and A. Star Angew. Chem. Int. Ed. 47, 6550-6570, 2008.

[2] X. Zhang et. al. Sensors, 13, 15159-15171, 2013. 